\title{
Spontaneous formation and optical manipulation of extended polariton condensates
}

\author{
E. Wertz ${ }^{1}$, L. Ferrier ${ }^{1}$, D. D. Solnyshkov ${ }^{2}$, R. Johne ${ }^{2}$, D. Sanvitto ${ }^{3}$, A. Lemaître' ${ }^{1}$, I. Sagnes ${ }^{1}$, \\ R. Grousson ${ }^{4}$, A. V. Kavokin ${ }^{5}$, P. Senellart ${ }^{1}$, G. Malpuech ${ }^{2}$ and J. Bloch ${ }^{1 \star}$
}

\begin{abstract}
Cavity exciton-polaritons ${ }^{1,2}$ (polaritons) are bosonic quasiparticles offering a unique solid-state system for investigating interacting condensates ${ }^{3-10}$. Up to now, disorder-induced localization and short lifetimes ${ }^{4,6,11}$ have prevented the establishment of long-range off-diagonal order ${ }^{12}$ needed for any quantum manipulation of the condensate wavefunction. In this work, using a wire microcavity with polariton lifetimes much longer than in previous samples, we show that polariton condensates can propagate over macroscopic distances outside the excitation area, while preserving their spontaneous spatial coherence. An extended condensate wavefunction builds up with a degree of spatial coherence larger than $\mathbf{5 0 \%}$ over distances $\mathbf{5 0}$ times the polariton de Broglie wavelength. The expansion of the condensate is shown to be governed by the repulsive potential induced by photogenerated excitons within the excitation area. The control of this local potential offers a new and versatile method to manipulate extended polariton condensates. As an illustration, we demonstrate synchronization of extended condensates by controlled tunnel coupling ${ }^{13,14}$ and localization of condensates in a trap with optically controlled dimensions.
\end{abstract}

Modern semiconductor technology allows the realization of nanostructures where both electronic and photonic states undergo quantum confinement. In particular in semiconductor microcavities, excitons confined in quantum wells and photons confined in a Fabry-Perot resonator can enter the light-matter strong coupling regime. This gives rise to the formation of cavity polaritons, mixed exciton-photon states that obey bosonic statistics ${ }^{2}$. The polariton dispersion presents a sharp energy minimum close to the states with zero in-plane wave vector $(k=0)$ with an effective mass $m^{*}$ three orders of magnitude smaller than that of the bare quantum well exciton. Recently, polariton Bose-Einstein condensation ${ }^{3-10}$ (BEC) and related effects such as vortices ${ }^{15,16}$ or superfluid ${ }^{17-19}$ behaviour have been reported at unprecedented high temperatures. As a result of their finite lifetime, cavity polaritons are a model system to investigate dynamical BEC (refs 20,21), also referred to as a polariton laser effect, with a technological control of the resonator geometry and the polariton lifetime. In previously reported polariton laser systems, the cavity lifetime and the photonic disorder prevented the build-up of extended condensates needed for the realization of polariton circuits ${ }^{22,23}$. The measured coherence length ranged at best from 10 to $20 \mu \mathrm{m}$ (refs 4,6,11,24), a few times the polariton thermal de Broglie wavelength.

Here, we report on the spontaneous formation of extended polariton condensates with a spatial coherence extending over 50 times the thermal de Broglie wavelength. These condensates, made a
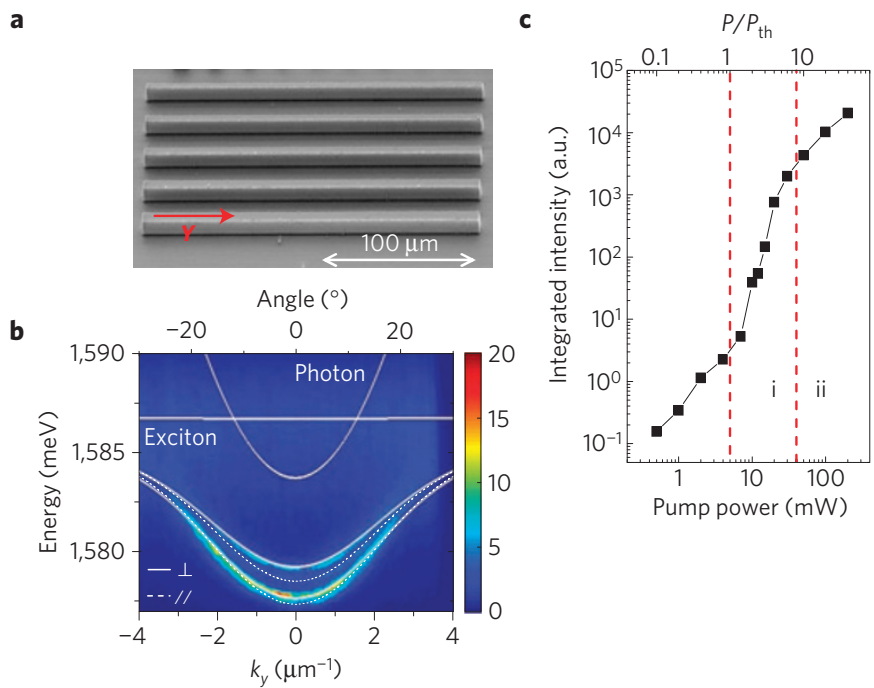

Figure 1 | Cavity polaritons confined in a microwire cavity. a, Scanning electron micrograph of the array of cavity wires. $\mathbf{b}$, Emission of a single wire as a function of detection angle, in a linear coloured scale, measured below threshold $\left(P=0.2 P_{\text {th }}\right)$ : such far-field measurements give direct information about the polariton distribution in $k_{y}$ space because the emission angle $\theta$ of a polariton state with energy $E$ is linked to $k_{y}$ by $E \sin (\vartheta)=\hbar c k_{y}$. Also shown with white lines are the uncoupled exciton and photon dispersion and the 1D polariton sub-bands. Notice that because of strain, these sub-bands are linearly polarized (parallel or perpendicular to the wire as indicated). The polariton population is broadly distributed along the observed sub-bands. c, Emission intensity measured on the whole wire and integrated over all $k$ values as a function of the excitation power. The excitation range for the appearance of one (respectively, several) polariton condensate is indicated by the letter i (respectively, ii). For $\mathbf{b}$ and $\mathbf{c}, L_{x}=3.5 \mu \mathrm{m}$ and $\delta=-3 \mathrm{meV}$.

of a quantum degenerated light-matter state, are strongly out of equilibrium, thus deeply differing from atomic BEC. Spatial control of such extended condensates is demonstrated, opening the way to a new range of physical phenomena. A large Rabi splitting ( $15 \mathrm{meV})$ microcavity of high optical quality is used, with a cavity photon lifetime of around $15 \mathrm{ps}$, at least 5 times larger than in previous reports of polariton condensation (see the Methods section). To manipulate the condensate wavefunctions, we use one-dimensional (1D) cavities defined by $200-\mu \mathrm{m}$-long microwires with a width of between 2 and $4 \mu \mathrm{m}$ (Fig. 1a). Such a small transverse wire dimension

\footnotetext{
'Laboratoire de Photonique et de Nanostructures, LPN/CNRS, Route de Nozay, 91460, Marcoussis, France, ${ }^{2}$ LASMEA, Clermont Université, Université Blaise Pascal, CNRS, 24 av des Landais, Aubiere, 63177, France, ${ }^{3}$ Departamento de Fisica de Materiales, Universidad Autonoma de Madrid, Madrid 28049 , Spain, ${ }^{4}$ Institut des NanoSciences de Paris, CNRS UMR 7588, Université P. et M. Curie, Campus Boucicaut, 140 Rue de Lourmel, 75015 Paris, France, ${ }^{5}$ Physics and Astronomy School, University of Southampton, Highfield, Southampton SO17 1BJ, UK. *e-mail: jacqueline.bloch@lpn.cnrs.fr.
} 

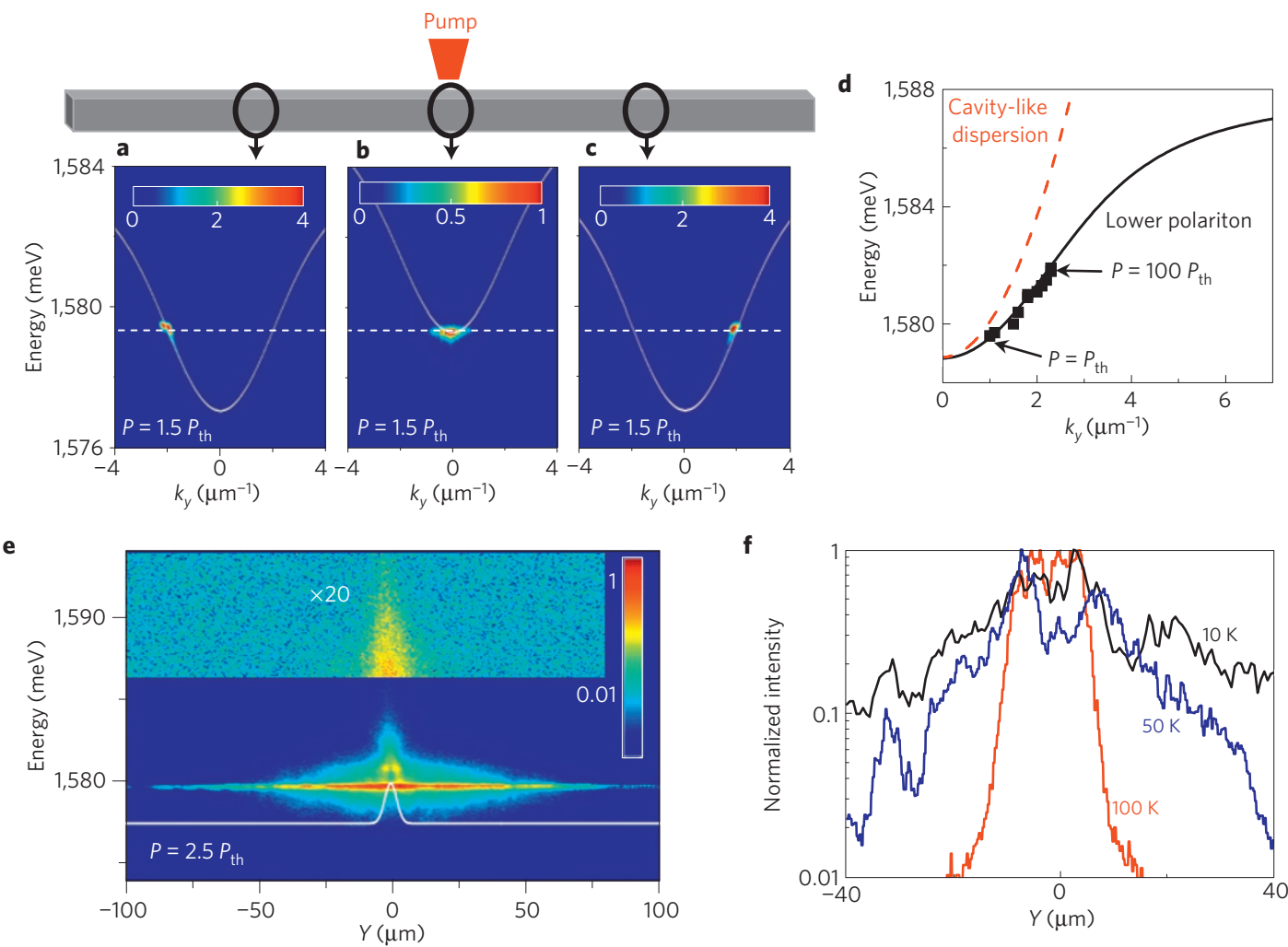

Figure 2 | Condensation and spatial spreading of cavity polaritons in microwires. a,c, Far-field emission above threshold $\left(P=1.5 P_{\text {th }}\right)$ of a $30 \mu \mathrm{m}$ wire section located on either side of the excitation area. $\mathbf{b}$, Far-field emission for $P=1.5 P_{\text {th }}$ of a $10 \mu \mathrm{m}$ wire section centred on the excitation spot. In $\mathbf{a}-\mathbf{c}$, the white line shows the polariton dispersion. $\mathbf{d}$, Measured energy of the condensate as a function of the condensate wave vector; also shown are the calculated polariton dispersion and photon-like dispersion. e, Real-space intensity distribution along the wire in a coloured logarithmic scale measured above threshold $\left(P=2.5 P_{\text {th }}\right)$ : condensed polaritons spread over the whole wire whereas higher-energy excitons remain in the excitation area (magnified top panel); the white line shows the optically induced potential. $L_{x}=3.5 \mu \mathrm{m}$ and $\delta=-3 \mathrm{meV}$. f, Intensity profile measured along the wire above threshold for various temperatures.

a
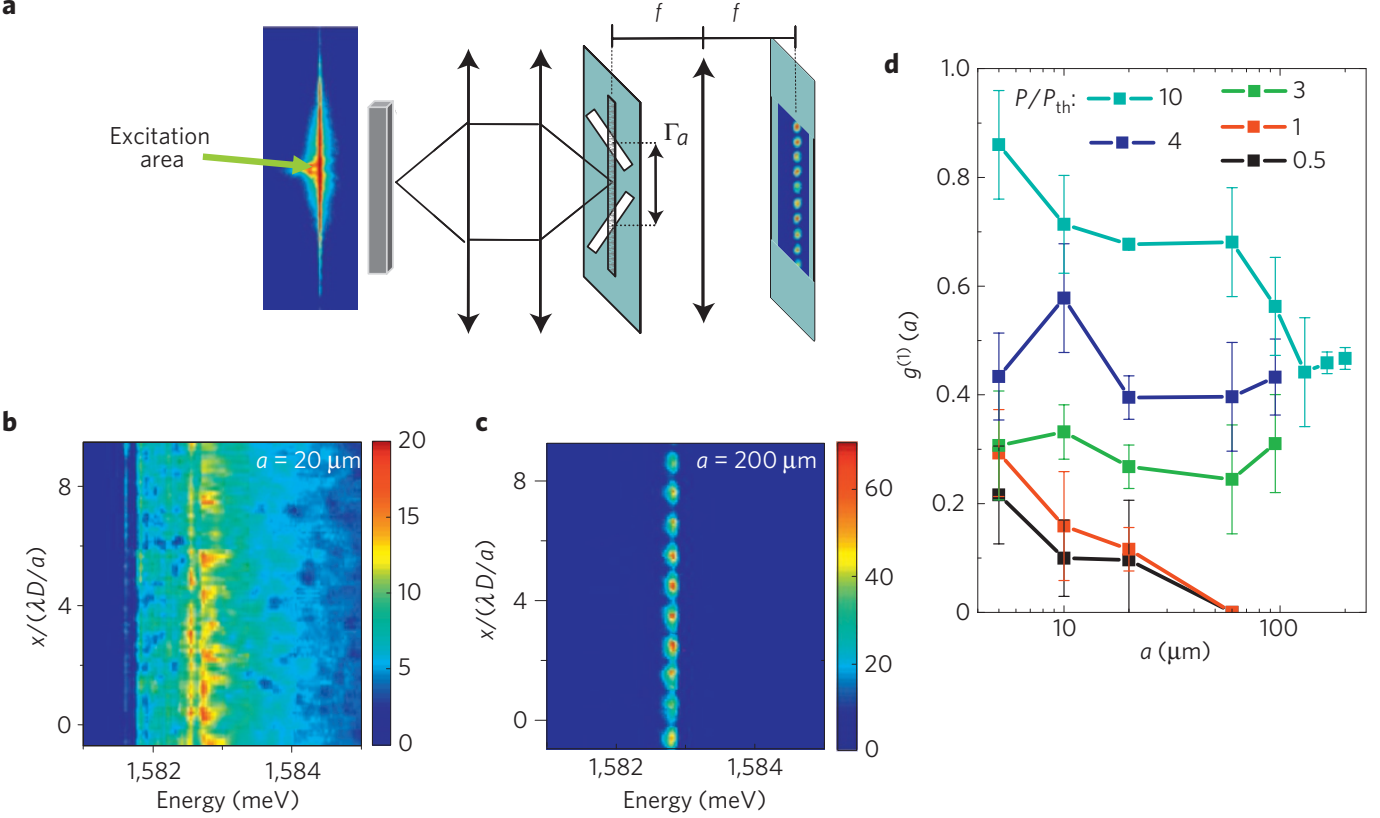

Figure 3 | Macroscopic off-diagonal long-range order. a, Schematic of the double-slit interferometry set-up for spatial coherence measurements: the interference pattern measured at infinity is projected onto the entrance slit of the spectrometer with a lens of focal length $f$. $\mathbf{b}$, Interference patterns for a distance $a=20 \mu \mathrm{m}$ between the two slits measured below threshold $\left(P=0.5 P_{\mathrm{th}}\right)$. c, Interference of the emission of the two wire ends ( $\left.a=200 \mu \mathrm{m}\right)$ measured above threshold $\left(P=10 P_{\text {th }}\right)$. d, First-order spatial coherence deduced from the fringe visibility measured for various excitation powers as a function of $a$. The error bars have been estimated considering the intensity fluctuations on the interference patterns. $L_{x}=3.5 \mu \mathrm{m}$ and $\delta=-2 \mathrm{meV}$. 


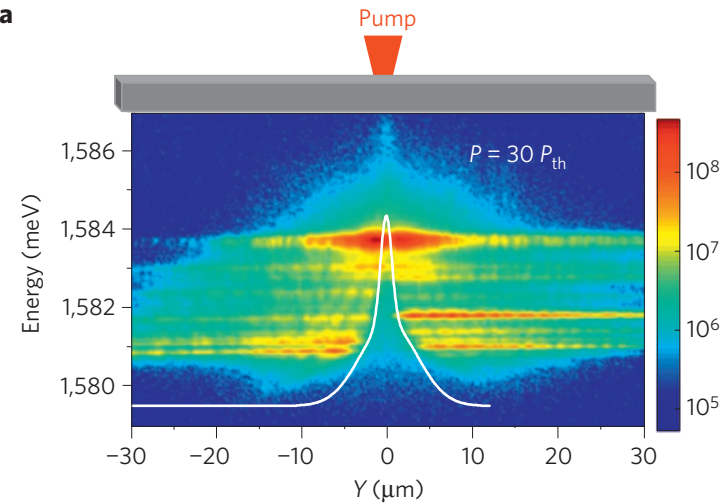

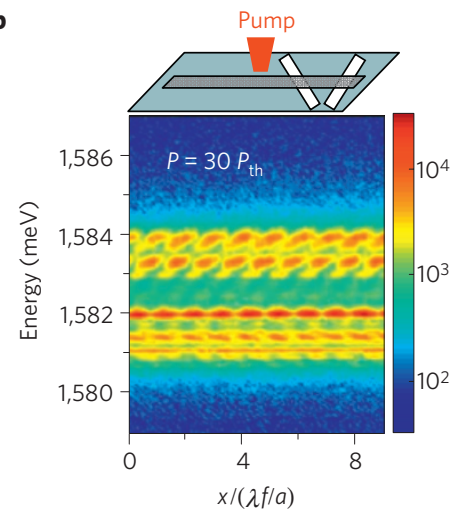

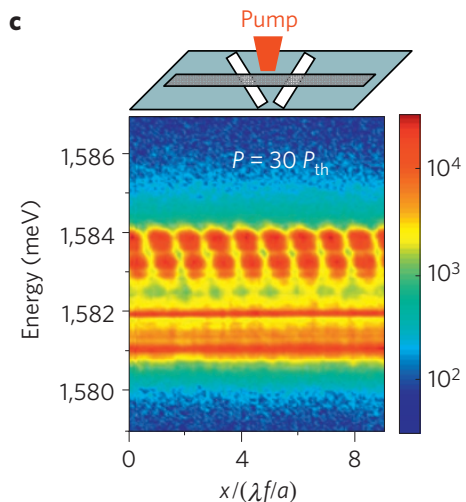

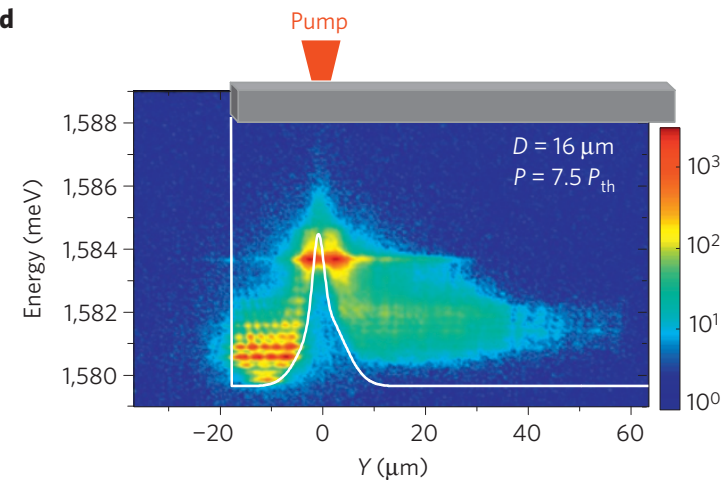

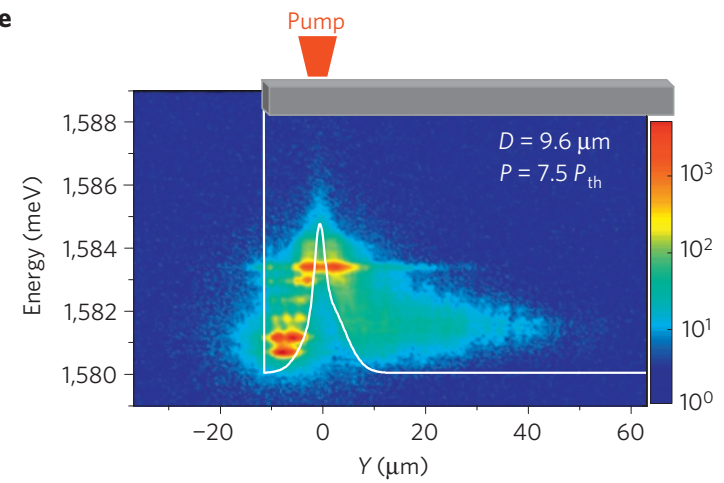

Figure 4 | Manipulation of the condensate wavefunction. We illustrate here how the condensate wavefunctions can be optically manipulated using the repulsive potential within the excitation spot. a, Emission intensity measured along the wire for an excitation power $P=30 P_{\text {th }}$; polariton relaxation induces the build-up of several delocalized condensates at lower energy. b,c, Spatial coherence measurements with $a=30 \mu \mathrm{m}$ using two slits on the same side (b) or on each side (c) of the excitation area: tunnel coupling through the central barrier allows synchronization of each side of the condensates for the highest-energy states. $\mathbf{d}, \mathbf{e}$, Trapping of the polariton condensates when approaching the laser spot to the wire end $\left(P=7.5 P_{\text {th }}\right)$. The wavefunctions of the discrete trapped states are directly imaged. The solid lines represent the barrier in the excitation area: we calculate the Coulomb potential induced by photoinjected excitons using a spatial distribution corresponding to the observed potential. a-c, $L_{x}=2.5 \mu \mathrm{m}$ and $\delta=-3 \mathrm{meV}$. $\mathbf{d}$ and $\mathbf{e}, L_{x}=3.5 \mu \mathrm{m}$ and $\delta=-2 \mathrm{meV}$. All images are plotted in a coloured log scale.

induces a lateral quantum confinement of polaritons and the formation of $1 \mathrm{D}$ sub-bands $\mathrm{s}^{25}$ (see Fig. $1 \mathrm{~b}$ for a $3.5 \mu \mathrm{m}$ wire).

Polaritons are generated optically using a non-resonant excitation provided by a single-mode continuous-wave laser beam focused down to a $2 \mu \mathrm{m}$ size spot at normal incidence. The laser energy is tuned to one of the reflectivity dips of the Bragg mirrors (typically $100 \mathrm{meV}$ above the lower polariton branch). At low excitation power, the far-field emission (resolved in $k$ space) shows a polariton population along several $1 \mathrm{D}$ branches (Fig. 1b). When raising the excitation power, a strong and abrupt increase of the overall wire emission intensity is observed, as shown in Fig. 1c. Spatially resolved measurements above threshold (see Fig. 2b) show that a polariton condensate is spontaneously formed within the excitation spot at the lowest-energy polariton state, close to $k_{y}=0$ (ref. 10). This condensate undergoes a local blueshift $E_{\mathrm{b}}$ owing to repulsive interaction with the high-density exciton cloud photogenerated in the excitation area. As there are no photogenerated excitons outside the small pump area, this blueshift is spatially limited and creates a force tending to expel polaritons from the excitation area: the polariton condensate undergoes a lateral acceleration and acquires a finite in-plane wave vector ${ }^{26}$. The far-field emission measured on each side of the excitation spot indicates the spontaneous generation of condensed polaritons with well-defined wave vectors given by $k_{y}= \pm \sqrt{2 m^{*} E_{\mathrm{b}}} / \hbar$ when approximating the polariton dispersion by a parabola ${ }^{26}$. Note that at high excitation powers, pair scattering of two polaritons in the pump spot into two polaritons exiting in the left and right directions at the same energy and opposite $k$ could also contribute to the observed feature. As the excitation power is increased, $E_{\mathrm{b}}$ and consequently $k_{y}$ increases, perfectly following the polariton dispersion (see Fig. $2 \mathrm{~d}$ ). The real-space image of the wire (Fig. 2e) shows that this finite in-plane wave vector leads to the propagation and expansion of the polariton condensate far on both sides of the excitation area. Energy analysis of the emission demonstrates that this extended condensed phase coexists with the cloud of uncondensed polaritons and excitons at higher energy, which remains in the close vicinity of the excitation spot (see the magnified upper panel of Fig. 2e).

To demonstrate the spatial coherence of the extended condensate, we carry out a Young slit interferometry ${ }^{11,27}$ experiment (Fig. 3a). An image of the photonic wire, magnified by a coefficient $\Gamma$, is formed in the plane of a screen, pierced with two tilted slits. $\Gamma$ is equal to 50 (respectively, 5) when probing values of a smaller (respectively, larger) than $30 \mu \mathrm{m}$. The spatial coherence between two small areas (with dimensions of the order of $0.5 \mu \mathrm{m}$ (respectively, $5 \mu \mathrm{m}$ )) symmetrically located at a distance $a / 2$ on each side of the excitation spot is probed. Figure $3 b, c$ shows interference patterns recorded below and above threshold. Below threshold, the emission is spectrally broad and interference fringes are barely visible. In contrast, above threshold pronounced fringes are observed with high visibility even for $a$ as large as $200 \mu \mathrm{m}$. The first-order spatial coherence $g^{1}(a)$ can be deduced from these interference patterns using: $g^{1}(a)=$ $U(a)\left(\left\langle I_{1}(r)\right\rangle+\left\langle I_{2}(r)\right\rangle\right) /\left(2 \sqrt{\left\langle I_{1}(r)\right\rangle\left\langle I_{2}(r)\right\rangle}\right)$, where $\left\langle I_{i}(r)\right\rangle$ is the average intensity measured with a single slit at position $r$ in the projection plane. $U(a)=\left(\langle I(r)\rangle_{\max }-\langle I(r)\rangle_{\min }\right) /\left(\langle I(r)\rangle_{\max }+\langle I(r)\rangle_{\min }\right)$ is the contrast between the maximum and minimum average 
intensities measured with both slits. Figure $3 \mathrm{~d}$ shows the measured $g^{1}(a)$ for several excitation powers. Below threshold, the coherence is $20 \%$ close to the excitation spot $(a=5 \mu \mathrm{m})$, and rapidly goes to zero for distances above $a=20 \mu \mathrm{m}$. Just above threshold, the coherence starts to increase and extends over a larger distance. Finally, for excitation powers exceeding the threshold by a factor of 3 or more, high spatial coherence is found all along the wire. This measurement demonstrates the formation of a coherent polariton wavefunction over the whole wire, a key feature proving the spontaneous build-up of macroscopic off-diagonal long-range order in the whole system ${ }^{12}$.

Further evidence of the coherence is obtained when approaching the laser beam at a distance $D$ from the wire end (see Supplementary SI). An interference pattern between waves travelling back and forth from the wire end is visible in the emission intensity along the wire.

In the following, we show that under higher excitation powers, several polariton condensates can coexist and be optically manipulated in a $1 \mathrm{D}$ cavity. Indeed, at high excitation powers, polariton-polariton interactions become efficient close to the excitation area, and induce polariton scattering towards lowerenergy states (see Supplementary SII). Several extended condensates appear on both sides of the excitation spot, which acts as a potential barrier (see Fig. 4a). Young slit interferometry carried out within either side of the pumping area demonstrates that each of these condensates shows off-diagonal long-range order (see Fig. 4b). Probing the spatial coherence between condensates located on opposite sides of the excitation spot indicates various regimes. As shown in Fig. 4c, low-energy condensates present no spatial coherence between the left and right side of the spot. Indeed, at these lowest energies, the potential barrier is thick enough to prevent tunnel coupling between the two sides. On the contrary, for the highest-energy condensates, a small signal in the central barrier demonstrates the tunnel coupling between the right and left part of the condensate. This tunnel coupling is responsible for phase locking of the two spatially separated condensates ${ }^{13,14}$. As a result, high spatial coherence all along the wire is shown for these states (see Fig. 4c). A simple estimation of polariton tunnelling efficiency through a $2 \mu \mathrm{m}$ potential barrier fully confirms this interpretation (see Supplementary SIII): when reducing the polariton energy by $1 \mathrm{meV}$ below the potential barrier, the tunnelling is reduced by three orders of magnitude. Thus, our experiments show that the repulsive potential created by optical pumping under the excitation spot can be used to generate extended condensates synchronized by controlled tunnel coupling, which opens the way to the realization of polariton Josephson junctions.

The simultaneous observation of condensation on several discrete states is the signature of the dynamical condensation regime in this strongly out-of-equilibrium 1D system: the build-up of a macroscopic population for certain states results from the complex interplay between scattering rates, energy conservation and lifetime of the polariton states. Control of these final states can be obtained by bringing the laser spot to the wire end: an energy trap with well-defined quantized states can be formed as illustrated in Fig. 4d,e. This trap is delimited on one side by the wire end (which acts as an infinite potential barrier) and on the other by the adjustable barrier defined by the excitation spot. 0D condensates are formed as indicated by the wavefunctions directly imaged in the intensity distribution within the trap.

As compared with previously reported techniques to spatially confine polariton condensates $5,7,28,29$, the size and height of the trap can be continuously controlled by changing both the excitation power and the position of the spot. Controlling both the propagation and the wavefunction of a polariton condensate opens the way to the realization of polariton circuits, a first step towards high-speed all-optical information processing ${ }^{22}$.
Finally let us highlight that the macroscopic propagation we report here is a unique feature of polariton condensates. Indeed we could obtain regular photon lasing in the very same wires at temperatures above $60 \mathrm{~K}$ (see Fig. $2 \mathrm{f}$ and Supplementary SIV): in this photon lasing regime, the coherent emission is limited to the excitation area without any propagation feature. Population inversion in the excitation spot induces a change of refractive index and results in gain-induced confinement within the excitation spot. Thus in the present work, we observe opposite behaviour between polariton condensation and photon lasing. The macroscopic propagation we observe for polariton condensates is intrinsically linked to their light-matter nature, as it is driven by Coulomb interactions undergone by their excitonic part. When going to a purely photon laser, this propagation feature fully disappears.

\section{Methods}

The photonic wires are fabricated using electron-beam lithography and reactive ion etching from the cavity sample described in ref. 10. Microphotoluminescence is measured at $10 \mathrm{~K}$ on a single wire using a single-mode continuous Ti:sapphire laser. Far-field (respectively, near-field) spectroscopy is carried out by projecting the Fourier plane (respectively, the image plane) of the microscope objective used to collect the emission on the entrance slit of a monochromator. The signal is then detected by a nitrogen-cooled CCD (charge-coupled device) camera. The sample shows a Rabi splitting of $15 \mathrm{meV}$. The exciton-photon detuning is defined as $\delta=E_{C}\left(k_{y}=0\right)-E_{X}\left(k_{y}=0\right)$, where $E_{C}\left(k_{y}=0\right)$ and $E_{X}\left(k_{y}=0\right)$ are the cavity mode and the exciton energy for $k_{y}=0$. Below threshold, the measured photoluminescence linewidth of the lower polariton and exciton lies around $150 \mu \mathrm{eV}$ and $4 \mathrm{meV}$, respectively.

Received 3 April 2010; accepted 9 July 2010; published online 29 August 2010

\section{References}

1. Weisbuch, C. et al. Observation of the coupled exciton-photon mode splitting in a semiconductor quantum microcavity. Phys. Rev. Lett. 69, 3314-3317 (1992)

2. Kavokin, A., Baumberg, J. J., Malpuech, G. \& Laussy, F. P. Microcavities (Oxford Univ. Press, 2007).

3. Deng, H. et al. Condensation of semiconductor microcavity exciton polaritons. Science 298, 199-202 (2002).

4. Kasprzak, J. et al. Bose-Einstein condensation of exciton polaritons. Nature 443, 409-414 (2006).

5. Balili, R. et al. Bose Einstein condensation of microcavity polaritons in trap. Science 316, 1007-1010 (2007).

6. Lai, C. W. et al. Coherent zero-state and $\pi$-state in an exciton-polariton condensate array. Nature 450, 529-532 (2007).

7. Bajoni, D. et al. Polariton laser using single micropillar GaAs-GaAlAs semiconductor cavities. Phys. Rev. Lett. 100, 047401 (2008).

8. Christopoulos, C. et al. Room-temperature polariton lasing in semiconductor microcavities. Phys. Rev. Lett. 98, 126405 (2007).

9. Christmann, G. et al. Room temperature polariton lasing in a GaN/AlGaN multiple quantum well microcavity. Appl. Phys. Lett. 93, 051102 (2008).

10. Wertz, E. et al. Spontaneous formation of a polariton condensate in a planar GaAs microcavity. Appl. Phys. Lett. 95, 051108 (2009).

11. Deng, H. et al. Spatial coherence of polaritons condensates. Phys. Rev. Lett. 99, 126403 (2007)

12. Penrose, O. \& Onsager, L. Bose Einstein condensation and liquid Helium. Phys. Rev. 104, 576-584 (1954).

13. Baas, A. et al. Synchronized and desynchronized phases of exciton-polariton condensates in the presence of disorder. Phys. Rev. Lett. 100, 170401 (2008).

14. Wouters, M. Synchronized and desynchronized phases of coupled nonequilibrium exciton-polariton condensates. Phys. Rev. B 77, 121302(R) (2008).

15. Lagoudakis, K. G. et al. Quantized vortices in an exciton-polariton condensate. Nature Phys. 4, 706-710 (2008).

16. Lagoudakis, K. G. et al. Observation of half-quantum vortices in an exciton-polariton condensate. Science 326, 974-976 (2009).

17. Usonomiya, S. et al. Observation of Bogoliubov excitations in exciton-polaritons condensates. Nature Phys. 4, 700-705 (2008).

18. Amo, A. et al. Collective fluid dynamics of a polariton condensate in a semiconductor microcavity. Nature 457, 291-295 (2009).

19. Amo, A. et al. Superfluidity of polaritons in semiconductor microcavities. Nature Phys. 5, 805-810 (2009).

20. Kavokin, A., Malpuech, G. \& Laussy, F. P. Polariton laser and polariton superfluidity in microcavities. Phys. Lett. A 306, 187-199 (2003). 
21. Keeling, J. et al. Polariton condensation with localized excitons and propagating photons. Phys. Rev. Lett. 93, 226403 (2004).

22. Liew, T. C. H., Kavokin, A. V. \& Shelykh, I. A. Optical circuits based on polariton neurons in semiconductor microcavities. Phys. Rev. Lett. 101, 016402 (2008).

23. Shelykh, I. A., Pavlovic, G., Solnyshkov, D. D. \& Malpuech, G. Proposal for a mesoscopic optical Berry-phase interferometer. Phys. Rev. Lett. 102, 046407 (2009).

24. Krizhanovskii, D. N. et al. Coexisting nonequilibrium condensates with long-range spatial coherence in semiconductor microcavities. Phys. Rev. B 80, 045317 (2009).

25. Dasbach, G. et al. Tailoring the polariton dispersion by optical confinement: Access to a manifold of elastic polariton pair scattering channels. Phys. Rev. B 66, 201201(R) (2002).

26. Wouters, M., Carusotto, I. \& Ciuti, C. Spatial and spectral shape of inhomogeneous nonequilibrium exciton-polariton condensates. Phys. Rev. B 77, 115340 (2008).

27. Dubin, F. et al. Macroscopic coherence of a single exciton state in an organic quantum wire. Nature Phys. 2, 32-35 (2005).

28. Idrissi Kaitouni, R. et al. Engineering the spatial confinement of exciton polaritons in semiconductors. Phys. Rev. B 74, 155311 (2006).
29. Sanvitto, D. et al. Exciton-polariton condensation in a natural two-dimensional trap. Phys. Rev. B 80, 045301 (2009).

\section{Acknowledgements}

This work was partly supported by the C'Nano Ile de France contract 'Sophiie2', by the ANR contract PNANO- 07-005 GEMINI, by the FP7 ITN 'Clermont4' (235114) and by the FP7 ITN 'Spin-Optronics' (237252).

\section{Author contributions}

E.W., L.F. and J.B. carried out the experiments and analysed the data. D.D.S., R.J., A.V.K. and G.M. theoretically studied the data. D.S. participated in some experiments. A.L. grew the sample, I.S. etched the microwires and R.G. gave fruitful advice for the Young slit experiments. P.S. did the electron-beam lithography, interpreted the data and wrote the paper. J.B. supervised the work and wrote the paper.

\section{Additional information}

The authors declare no competing financial interests. Supplementary information accompanies this paper on www.nature.com/naturephysics. Reprints and permissions information is available online at http://npg.nature.com/reprintsandpermissions.

Correspondence and requests for materials should be addressed to J.B. 